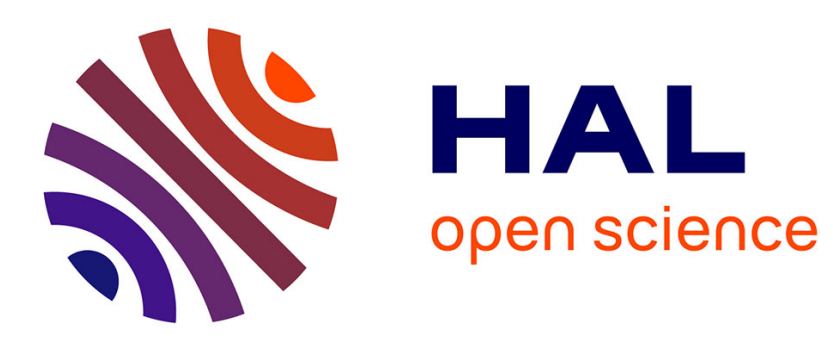

\title{
Shaped Continuous Parallel Plate Delay Lens With Enhanced Scanning Performance
}

François Doucet, Nelson J. G. Fonseca, Etienne Girard, Xavier Morvan, Laurent Le Coq, Hervé Legay, Ronan Sauleau

\section{To cite this version:}

François Doucet, Nelson J. G. Fonseca, Etienne Girard, Xavier Morvan, Laurent Le Coq, et al.. Shaped Continuous Parallel Plate Delay Lens With Enhanced Scanning Performance. IEEE Transactions on Antennas and Propagation, 2019, 67 (11), pp.6695-6704. 10.1109/TAP.2019.2924904 . hal-02391233

\section{HAL Id: hal-02391233}

\section{https://hal-univ-rennes1.archives-ouvertes.fr/hal-02391233}

Submitted on 4 Feb 2020

HAL is a multi-disciplinary open access archive for the deposit and dissemination of scientific research documents, whether they are published or not. The documents may come from teaching and research institutions in France or abroad, or from public or private research centers.
L'archive ouverte pluridisciplinaire HAL, est destinée au dépôt et à la diffusion de documents scientifiques de niveau recherche, publiés ou non, émanant des établissements d'enseignement et de recherche français ou étrangers, des laboratoires publics ou privés. 


\title{
Shaped Continuous Parallel Plate Delay Lens with Enhanced Scanning Performance
}

\author{
François Doucet, Nelson J. G. Fonseca, Senior Member, IEEE, Etienne Girard, Xavier Morvan, \\ Laurent Le Coq, Hervé Legay and Ronan Sauleau, Fellow, IEEE
}

\begin{abstract}
Shaped continuous parallel plate waveguide delay lenses are proposed in this paper. The previous configurations introduced by the authors displayed a residual side lobe imbalance, showing the limitations of elliptical delay lens profiles, derived from a starting bifocal constrained lens. Polynomial profiles are proposed here as a way to enhance the scanning performance and the pattern shape. Optimized configurations demonstrate a reduction of the phase aberration levels over a large scanning range $\left(\left[-30^{\circ}, 30^{\circ}\right]\right)$. The associated radiation patterns demonstrate lower and more balanced first side lobe

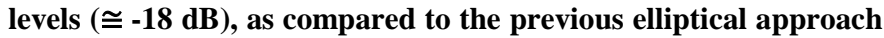
$(\cong-13.2 \mathrm{~dB})$. A validation has been proposed through the manufacturing and test of a prototype over the down-link Ka band ([27.5-31] GHz). Excellent radiation performance (HPBW, SLL) has been obtained experimentally over a wide angular range $\left(\left[-31.5^{\circ}, 31.5^{\circ}\right]\right)$, including low scanning loss and high radiation stability over the entire frequency range. High radiation efficiencies are demonstrated with this fully metallic design, particularly suitable for space applications. The mechanical simplicity offered by the concept is also quite attractive for low-cost multi-beam platforms.
\end{abstract}

Index Terms - Beamformer, constrained lens, geometrical optics (GO), ray tracing, multiple beam antennas, parallel plate waveguide (PPW)

\section{INTRODUCTION}

The increasing demand for broadband communication systems leads to the deployment of a large number of satellites, operating at very high frequencies and proposing multiple beam coverage to reuse efficiently the available bandwidth [1], [2]. Most of the antenna systems currently proposed are based on a complex design approach, explaining some efforts needed for the development of low-cost solutions, while maintaining stringent RF constraints. Simpler mechanical designs operating over a wide frequency range or possibly over up- and down-link bands while covering a wide angular sector are required.

A particular attention has been given recently to quasioptical beamformers as they provide scanning capabilities

This work was supported in part by the European Space Agency (ESA) and in part by Thales Alenia Space, France, in the frame of ESA's Networking/Partnering Initiative (NPI).

F. Doucet, X. Morvan, L. Le Coq and R. Sauleau are with Univ Rennes, CNRS, IETR (Institut d'Electronique et de Télécommunications de Rennes) UMR 6164, F-35000 Rennes, France (e-mail : fradoucet@gmail.com).

N. J. G. Fonseca is with the European Space Agency, 220 AG Noordwijk, The Netherlands (e-mail: nelson.fonseca@esa.int).

E. Girard and H. Legay are with the Research and Development Department, Thales Alenia Space, 31037 Toulouse, France (e-mail: etienne.girard@thalesaleniaspace.com). over a wide band of operation while saving cost in manufacturing. One of the simplest design, first described in the 50's, is the pillbox antenna [3] working with a two dimensional circular profile, limited by spherical phase aberrations. This pillbox configuration has been studied in several antenna designs (e.g., [4], [5], [6]) proposing a parabolic profile with one on-axis true focal point, resulting in phase aberrations which increase drastically with the scanning angle. To extend the scanning capabilities, Ruze [7] and Rotman [8] lenses were introduced, making use of discrete transmission lines connecting the outer lens contour and the radiating aperture. Nevertheless, the discretization performed for the aperture introduces some limitations. Most designs rely on a port spacing of half-a-wavelength, setting an upper limit on the operating frequency [9]. Wide band performance can be achieved using coaxial cables, enabling a very fine discretization of the lens but leading to more complex manufacturing and assembly processes, as well as degraded port-to-port coupling. The 2D Luneberg lenses [10] are also particularly attractive to target very large scanning performance, by synthetizing a graded refractive index within a parallel plate waveguide. Solutions using a periodic distribution of pins [11] or holes [12] were proposed, but their manufacturing is complex and becomes challenging at high frequencies. An alternative solution based on the geodesic lens, also known as the Rinehart-Luneburg lens [13] has been proposed in [14]. This approach proposes a significant reduction of the geodesic curve height, which is an inherent limitation of the concept, while making it more suitable for millimeter and submillimeter wave manufacturing constraints. Those solutions are nonetheless limited by their overall size $(f / D=1)$ due to the rotational symmetry properties.

A new concept of continuous parallel plate waveguide (PPW) lens-like beamformer has been recently introduced [15]. This beamformer (Fig. 1a) solution transforms a cylindrical wave, launched by one of the primary feeds (Fig. 1a), into a nearly plane wave exiting a radiating horn. The phase conversion is ensured by the use of a transversal ridge and cavity (Fig. 1b). The main beam direction $\theta_{2}$ is defined by the angular feed position $\theta_{1}$ along the focal curve (dotted line in Fig. 1c), with $\theta_{2}=\theta_{1}$. An experimental validation of the concept is reported in [15].

An analytical model [16], [17] has been developed based on geometrical optics (GO), which demonstrates good and timeefficient prediction of the proposed radiation properties when compared to full-wave analyses. This analytical model enabled more extensive optimizations and two methods were compared in [18], one based on phase-only error minimization and one on pattern constraints. All proposed optimized 


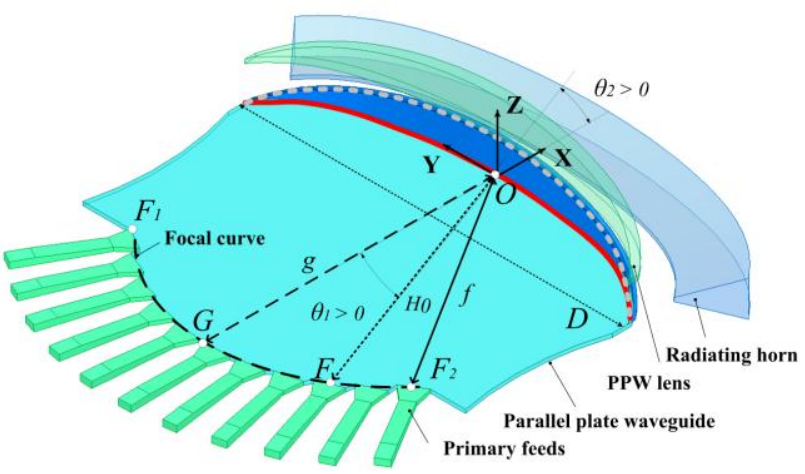

(a)

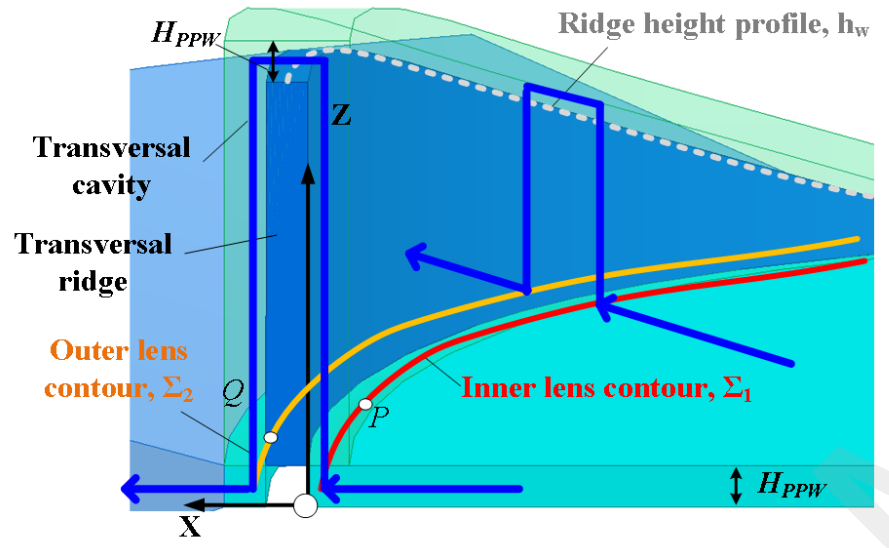

(b)

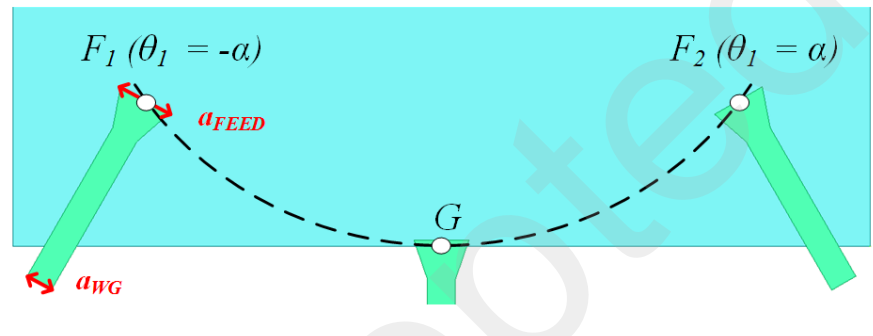

(c)

Fig 1. Continuous parallel plate waveguide (PPW) lens-like beamformer [17]: (a) 3D view, (b) zoom on the ridge and cavity parts, and (c) top view of the feeds along the focal curve. The electric field flow in the transversal cavity is illustrated by the blue arrows in Fig. 1b.

configurations display a residual side lobe imbalance, which appears as an inherent limitation of the considered elliptical profiles, derived from a simplified bifocal constrained lens model [19].

Alternative delay lens profiles were investigated with the aim to improve the pattern shape, and particularly reduce the side lobe levels, while maintaining the benefits of the original design, namely its mechanical simplicity and high efficiency. In this paper, shaped polynomial delay lens profiles (inner lens contour $\left(\Sigma_{1}\right)$ and ridge height profile $\left.\left(h_{w}\right)\right)$ are considered to increase the control on both phase and amplitude distributions

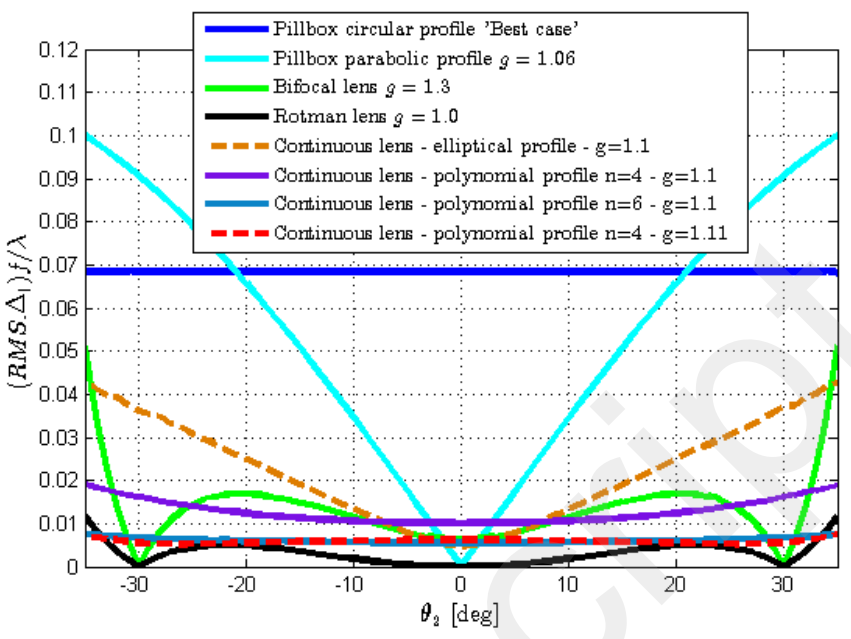

Fig 2. RMS value of the path length errors normalized to the wavelength $\lambda$ versus pointing angle $\theta_{2}$ for different lens models and different beamformer types, defined with the same diameter $D=10 \lambda$

along the outer lens contour $\left(\Sigma_{2}\right)$ and improve the radiation performance. The paper is organized as follows. The theoretical approach is presented in Section II, and optimized configurations are analyzed and compared with the results obtained in [18]. An experimental validation is presented in Section III and conclusions are drawn in Section IV.

\section{POLYNOMIAL SHAPED DELAY LENS - THEORETICAL APPROACH}

The design parameters used in this section are shown in Fig. 1a. For comparison purposes, the configuration considered here is the same as in [18]. The lens diameter and the focal distance are respectively set to $D=10 \lambda$ and $f=0.7 D$, where $\lambda$ is the wavelength in free space at the center frequency. The bifocal constrained lens approach, previously proposed as a starting point to design the continuous delay lens, is also considered here. Two focal points positions $F_{1}$ and $F_{2}$ are defined by their angular positions $\theta_{1}=\alpha=30^{\circ}, \theta_{1}=-\alpha=-30^{\circ}$ respectively, as shown in Fig. 1c. A point source located at each focal point produces beams pointing at $\theta_{2}=\theta_{1}$. Both focal points and the lens are symmetrical with respect to $x$-axis; as a consequence, only $F_{2}$ is considered in the optimization procedure. The feed points towards $O$, center of the delay lens, to ensure a suitable illumination. The feed horn aperture is equal to $1.5 \lambda$. The inner lens contour $\left(\Sigma_{1}\right)$ and the ridge height profile $\left(h_{w}\right)$ (Fig. 1b) are defined here by the following polynomial expressions

$$
x=\sum_{k=1}^{n} p_{k} y^{k}
$$

and,

$$
h_{w}=\sum_{k=1}^{n} q_{k} y^{k}-\min \left(\sum_{k=1}^{n} q_{k} y^{k}\right),
$$

where $x, y$ and $h_{w}$ are the coordinates along the different reference system axis, all normalized to the focal distance $f$. $p_{k}$ and $q_{k}$ are the $k^{t h}$ degree coefficients, where $1 \leq k \leq n, n$ 
defining the maximum degree of the polynomial functions. This formulation requires minor updates to the analytical model previously derived for elliptical profiles [12]. This approach, based on ray tracing, requires the knowledge of the local tangents (to apply Snell's laws), which are defined analytically for the differentiable functions considered here. The outer lens contour $\left(\Sigma_{2}\right)$ is defined as a translation of the inner contour along $x$-axis. In this theoretical approach, this translation value is small in terms of $\lambda$, without real impact on the radiation performance. Using the lens symmetry with respect to $x$-axis, only even order coefficients are considered. Those coefficients are optimization parameters for the beam associated to $F_{2}$. As presented in [18], a differential evolution (DE) metaheuristic algorithm ([20], [21]) is used to optimize the lens profiles. The latter are optimized for the feed located at $F_{2}$, the parameter $g=O G / f$ being used as an additional tuning parameter to enhance performance for any beam radiated in the scanning range $[-\alpha, \alpha]$ by adjusting the curvature of the focal arc. This approach is similar to the one used on previous designs derived from the simplified bifocal constrained lens model [19].

A phase-only optimization is first conducted and, as described in [18] where only elliptical profiles were considered, the objective is to minimize the path length errors $\Delta_{l}$ computed along the outer lens contour $\left(\Sigma_{2}\right)$, defined as

$$
\Delta_{l}=F_{2} P Q-F_{2} P Q\left[y_{Q}=0\right] .
$$

The difference in electrical path length is defined between any ray coming from $F_{1}$ (Fig. 1) and a central ray chosen as a reference, passing through the center of the lens $O$ on the outer lens contour $\left(\Sigma_{2}\right)$.

The root mean square of those errors serves as a cost function [18]. RMS values of the path length errors normalized to the wavelength $\lambda$ versus pointing angle $\theta_{2}$ are plotted in Fig. 2, for different lens models and different beamformer types, defined with the same diameter $D=10 \lambda$. All the configurations are identical, with $f=0.7 D$, expect for the initial pillbox design proposed by Rotman in [3], as the concept considers two concentric lens and focal curve profiles. In this case, the position of the circular focal curve, defining the best scanning performances, is proposed for the comparisons. As explained previously in the introduction, this approach is limited by spherical path length errors. This is clearly shown here with a constant RMS value (blue curve) over the scanning range considered. The parabolic profile approach (turquoise curve) demonstrates one true focal point with phase aberrations increasing strongly with the scanning angle $\theta_{2}$.

In our case, starting with $n=4$ (purple curve), a substantial reduction of the phase aberrations is observed for the beam associated to $F_{1}\left(\theta_{1}=\alpha=30^{\circ}\right)$, when compared to the previous optimized elliptical profiles (orange curve) [18]. Increasing the polynomial degree with $n=6$ or 10 the phase aberrations may be further reduced for $\theta_{2}=\alpha$, with some convergence observed which does not allow for a true focal point $(\mathrm{RMS}=0)$ as in the case of the Rotman lens (black curve) and bifocal constrained lens (green curve). Using $g=G O / f$ as an additional parameter for all the

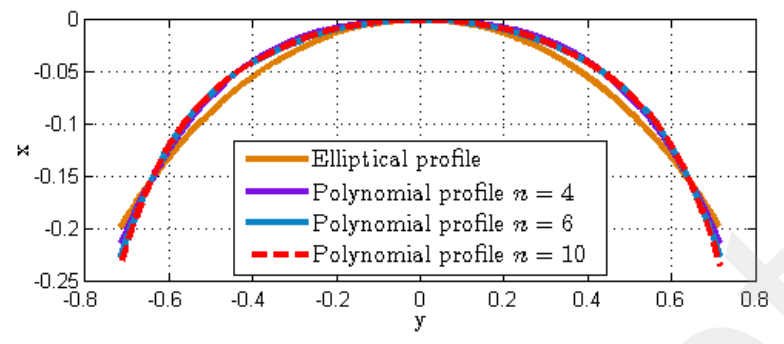

(a)

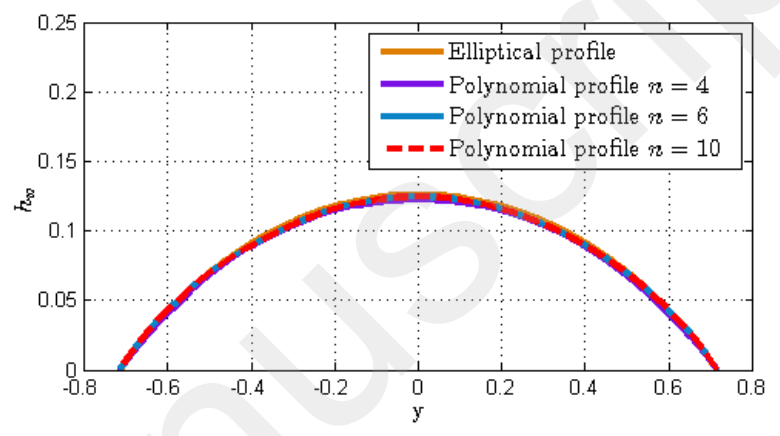

(b)

Fig 3. Comparison between elliptical and polynomial shapes of the PPW lens-like beamformer after phase-only optimization: (a) inner lens contour, (b) ridge height profile. Axis values are normalized to the focal distance $f$.

configurations, suitable values have been defined to minimize the maximum RMS level over the entire scanning range $[-\alpha, \alpha]$. The results obtained for $\mathrm{n}=6$ or 10 are almost identical, thus indicating that there is no benefit in increasing further the order of the polynomial functions.

Shapes of the inner lens contour $\left(\Sigma_{1}\right)$ and of the ridge height profile $\left(h_{w}\right)$ are plotted and compared in Fig. 3 for the various delay-lens configurations analyzed. The differences observed between optimized polynomial and elliptical shapes (Fig. 3a) are quite small but still significant in view of the RMS error reduction observed. This is obviously the consequence of the added degrees of freedom in the delay lens design which could not be achieved using the approach previously considered in [17].

The patterns radiated by the different GO models are plotted in Fig. 4a. Delay lenses with optimized elliptical profiles have a residual side lobe imbalance $(S L L=-13.2$ $\mathrm{dB}$ ), which is problematic for multiple beam satellite applications as this results in higher interference between beams. Optimized polynomial profiles demonstrate a lower and more balanced first side lobe level $(\cong-18 \mathrm{~dB})$. Interestingly, the radiation patterns are very similar for the three polynomial cases considered, indicating again that the further reduction of phase aberrations observed with $n=6,10$ has limited benefit on the first side lobes. A comparison with HFSS [23] is also proposed in Fig. 4b for the lens model with $n=10$. The HFSS model is composed of a PPW section closed by PML boundaries and excited by a single primary feed at the focal point $F_{2}$, the objective is to be closer as possible to the GO numerical model assumptions as described 


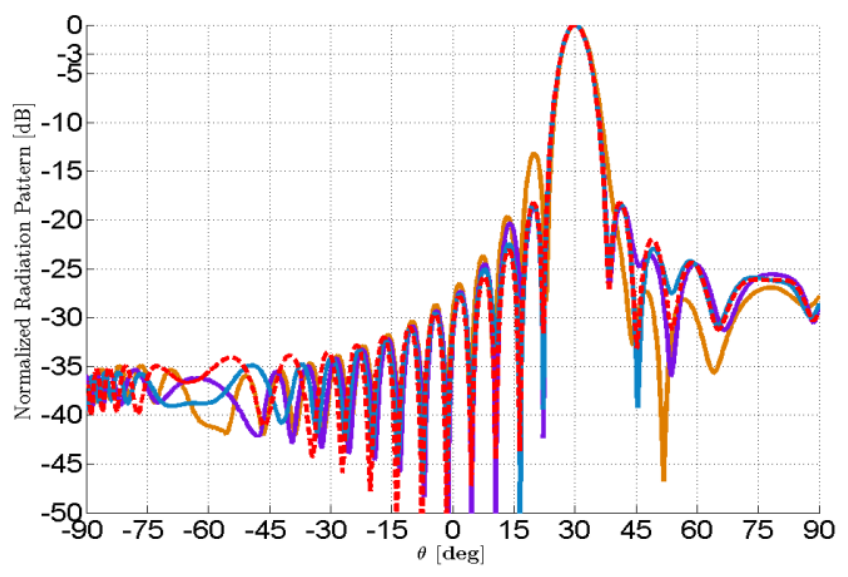

(a)

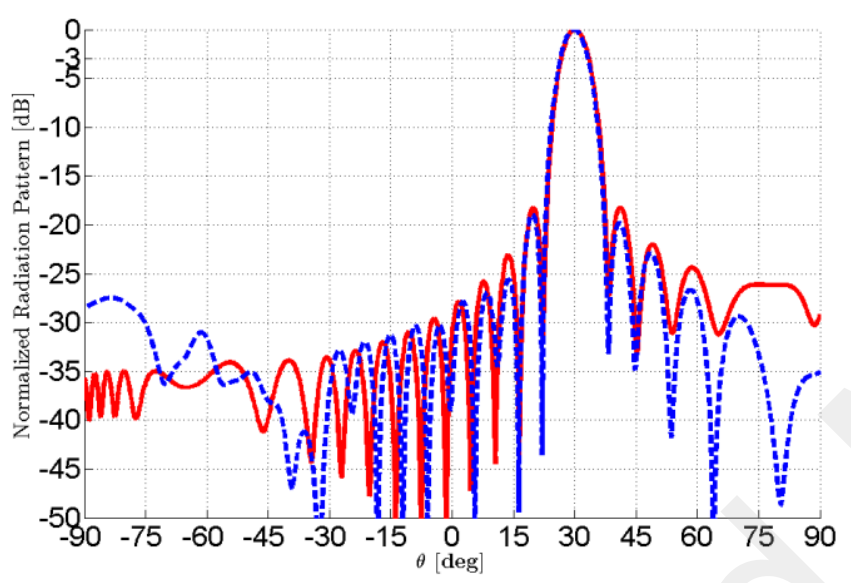

(b)

Fig 4. Radiation patterns of the PPW beamformer in H-plane for $\theta_{1}=\alpha$, at the central frequency: (a) GO models, (b) GO continuous lens model - phaseonly optimization (validation with HFSS) only considering $n=10$. The polynomial profiles coefficients are the following: $p=[-0.999,-0.597,0.0618,-0211,-0.223,0]$ and

$q=[-0.178,-0.031,-0.001,-0.029,-0.210,0]$.

in this paper. The pointing direction $\theta_{2}$, the half power beamwidth $\theta_{3 d B}$ and the first side lobe level $S L L$ equal $30^{\circ}$, $6.7^{\circ},-18.2 \mathrm{~dB}$ and $29.7^{\circ}, 6.6^{\circ},-18.9 \mathrm{~dB}$ respectively for the GO model and HFSS results. Both sets of data are in excellent agreement for levels of about 25 to $30 \mathrm{~dB}$ below the peak directivity.

As mentioned in previous publications [18], the amplitude distribution over $\Sigma_{2}$ and consequently the SLL may be impacted by the distribution of rays. For this reason, a patternbased optimization is also investigated. The same constraints as in [18] for a similar configuration $(f=0.7 D)$ are implemented: $\theta_{\text {target }}=30^{\circ}, \theta_{3 d B}=6.5^{\circ}$ and $S L L<-18 \mathrm{~dB}$, those values being derived from the ideal bifocal constrained lens model [17]. Several optimizations are repeated to target a $S L L$ as low as possible.

The optimized patterns are plotted in Fig. 5. This figure shows that the results are very similar to those obtained with the phase-only optimization procedure (Fig. 4a) for all values

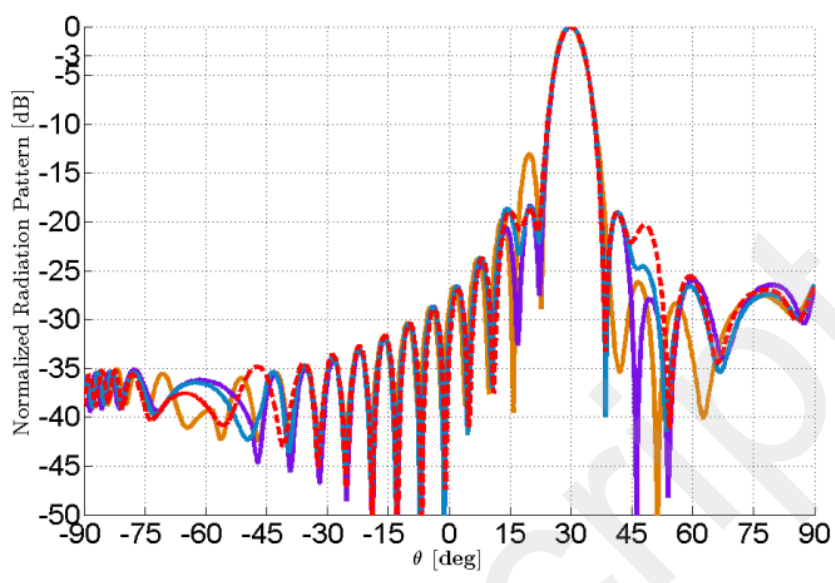

(a)

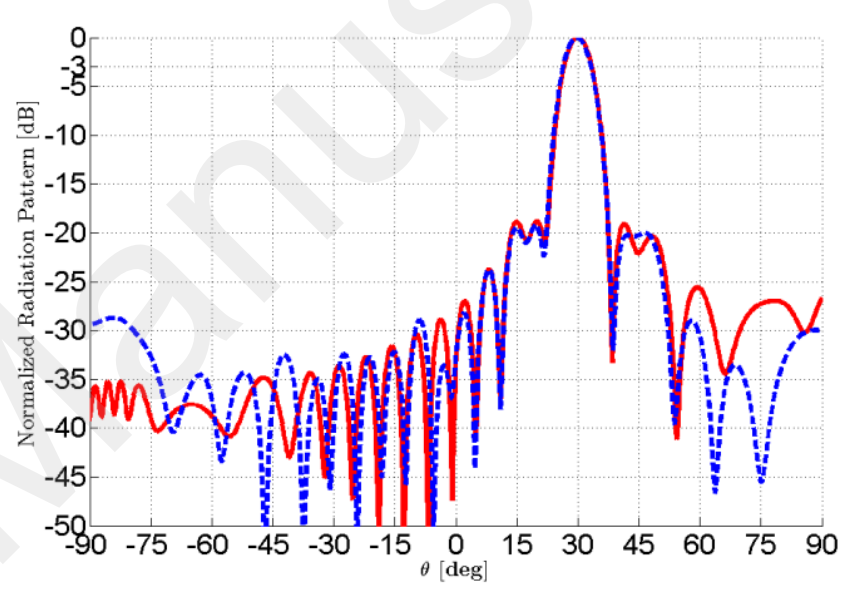

(b)

Fig 5. Radiation patterns of the PPW beamformer in H-plane for $\theta_{1}=\alpha$, at the central frequency: (a) GO models, (b) GO continuous lens model pattern optimization (validation with HFSS) only considering $n=10$. The polynomial profiles coefficients are the following: $p=[-0.912,0.378,-0.980,0.186,-0.269,0]$ and $q=[-0.129,-0.206,0.024,0.103,-0.243,0]$.

of $n$. A good agreement between HFSS [23] and the GO tool is maintained using this optimization method, as demonstrated in Fig. 5b. A marginal improvement on the SLL is observed (ranging from 0.5 to $1 \mathrm{~dB}$ ), indicating that the side lobes imbalance is mostly driven by phase aberrations.

The numerical results presented in this section clearly indicate the potential of the polynomial-shaped delay lens over the previously investigated elliptical-based lens designs. These results are validated in the following section through the manufacturing and test of a prototype.

\section{POlynomial Shaped DElay Lens Design AND EXPERIMENTAL VALIDATION}

\section{A. Design and Numerical Results}

The delay-lens prototype discussed in this section has been designed to work over the entire up-link Ka-band [27.5-31 $\mathrm{GHz}$ ] allocated to satellite communications. The lens diameter and the focal distance are equal to $D=20 \lambda$ and $f=0.7 D$ 


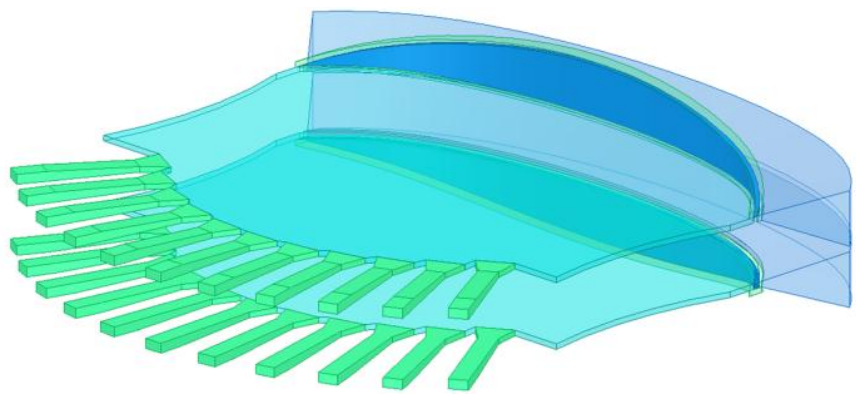

(a)

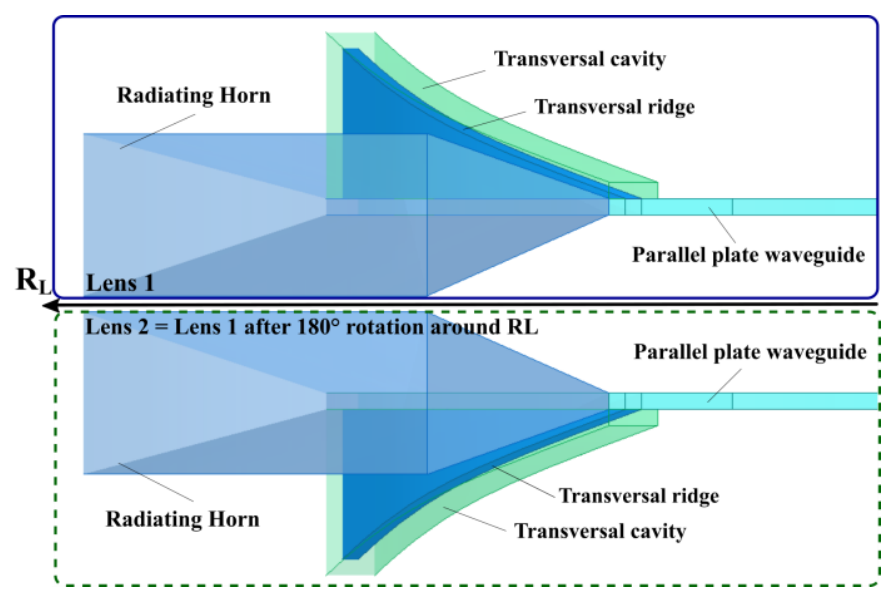

(b)

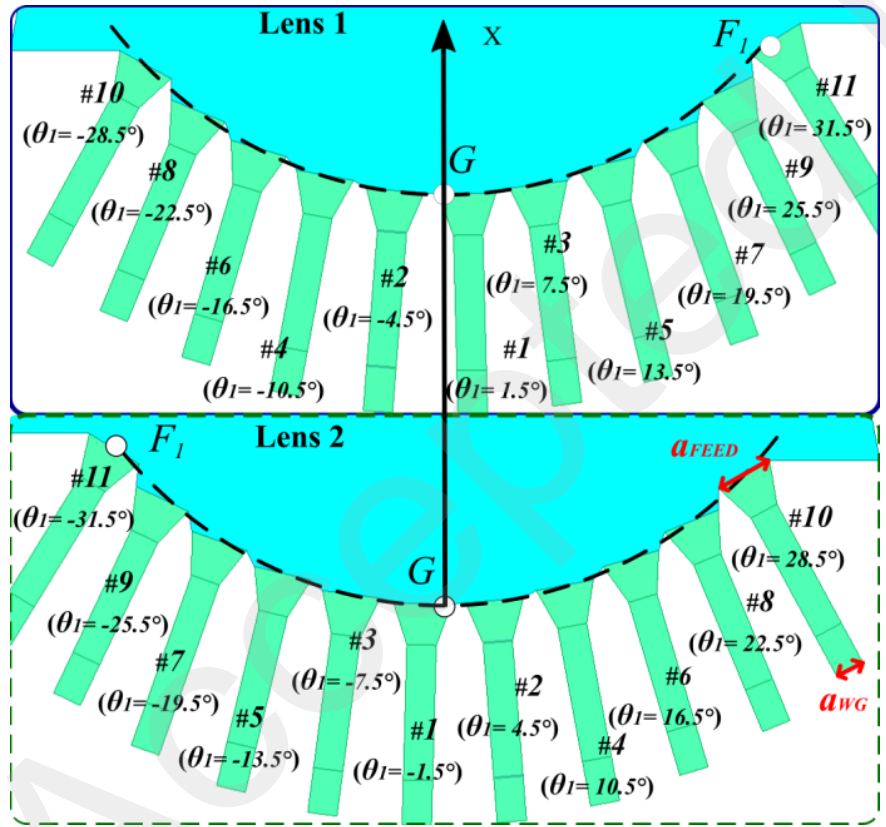

(c)

Fig 6. Antenna system to provide a complete coverage of 22 beams with a desired $3 \mathrm{~dB}$ cross-over level. (a) $3 \mathrm{D}$ view of the final configuration with two superimposed lenses, (b) side view with Lens 2 identical to Lens 1, defined with a $180^{\circ}$ rotation around $\mathrm{R}_{\mathrm{L}}$ and (c) $180^{\circ}$ rotation of the feed distribution obtained between Lens 1 and Lens 2.

respectively, where $\lambda=10.9 \mathrm{~mm}$ is the wavelength in free space at the lowest frequency of the operating band. The PPW

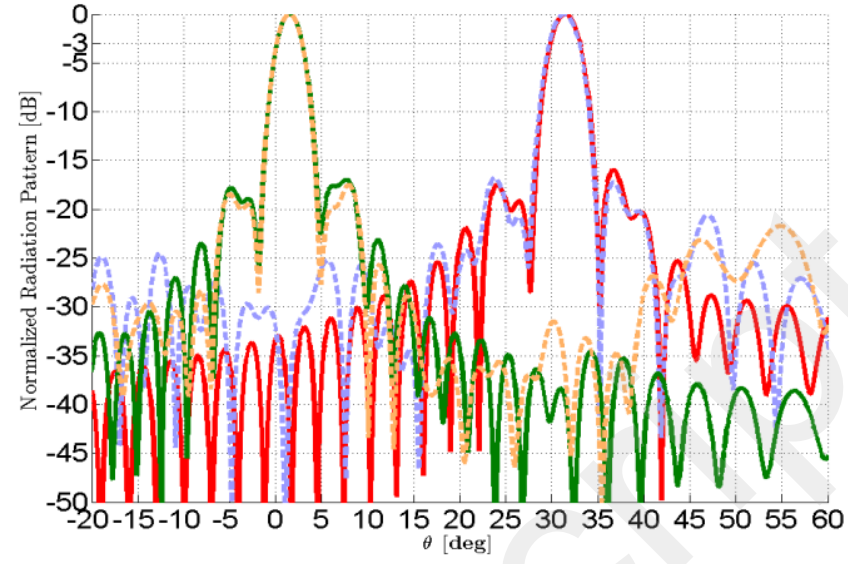

(a)

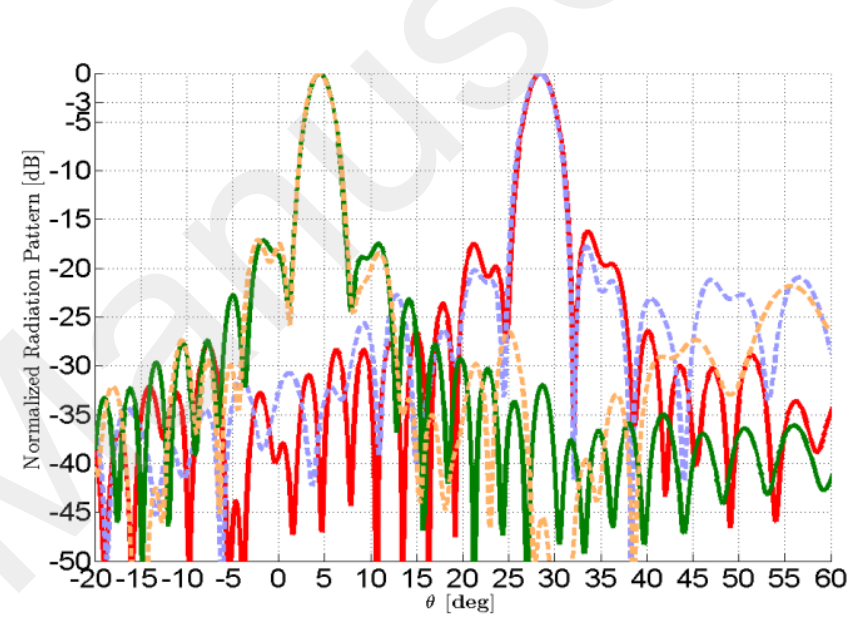

(b)

Fig 7. H-plane normalized radiation patterns of the PPW beamformer at $f_{0}=30 \mathrm{GHz}$. (a) Lens 1 - feeds \#11 $\left(\theta_{1}=31.5^{\circ}\right)$, \#1 $\left(\theta_{1}=1.5^{\circ}\right)$. (b) Lens 2 - feeds \#10 $\left(\theta_{1}=28.5^{\circ}\right), \# 2\left(\theta_{1}=4.5^{\circ}\right)$. GO model (continuous line) and final FEM simulation (dotted line).

cavity height is equal to $H_{P P W}=2 \mathrm{~mm}$, as only the fundamental TEM mode can propagate within the frequency band considered. The outer lens contour $\left(\Sigma_{2}\right)$ is defined as a translation of $2 \mathrm{~mm}$ of the inner lens contour $\left(\Sigma_{1}\right)$ along $x$ axis. The radiating horn length equals $30 \mathrm{~mm}$, defined along the local normal vector of the outer lens contour $\left(\Sigma_{2}\right)$, with a final height of $20 \mathrm{~mm}$.

Eleven feeds are distributed on a circular focal curve (Fig. $6 \mathrm{~b})$ with angular positions $\theta_{1}$ ranging from $-28.5^{\circ}$ to $31.5^{\circ}$ with a uniform angular step of $6^{\circ}$. The feeds dimensions are equal to $a_{W G}=7.556 \mathrm{~mm}$ and $a_{F E E D}=14 \mathrm{~mm}$. This configuration provides patterns with a beamwidth $\theta_{3 d B}=3^{\circ}$ while targeting minimum SLL, which requires an adequate edge taper and hence a minimum feed aperture. To provide the adequate cross-over level between adjacent beams (typically lower larger than $3 \mathrm{~dB}$ below the peak directivity), an antenna system made of two superimposed lenses is proposed, as shown in Fig. 6a. Both lenses are identical and symmetrically placed on both side of an axis $R_{L}$ (Fig. 6b). This lens stack provides 22 equally spaced beams with the desired cross-over 


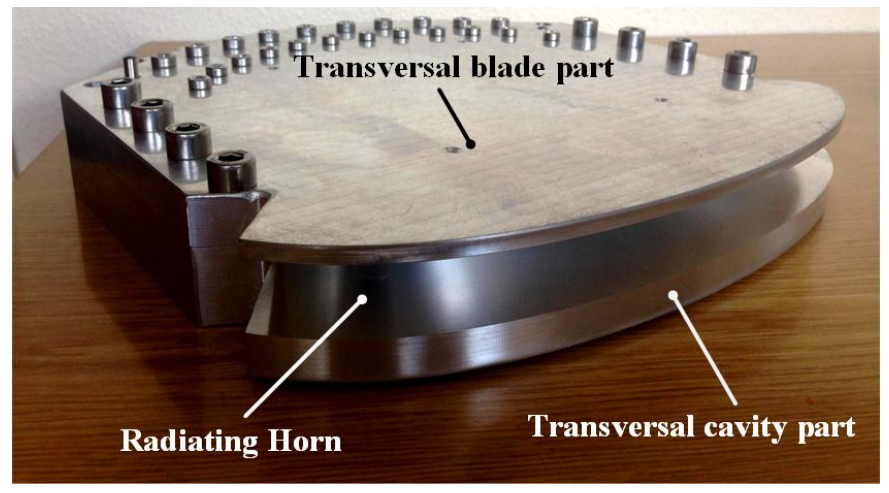

(a)

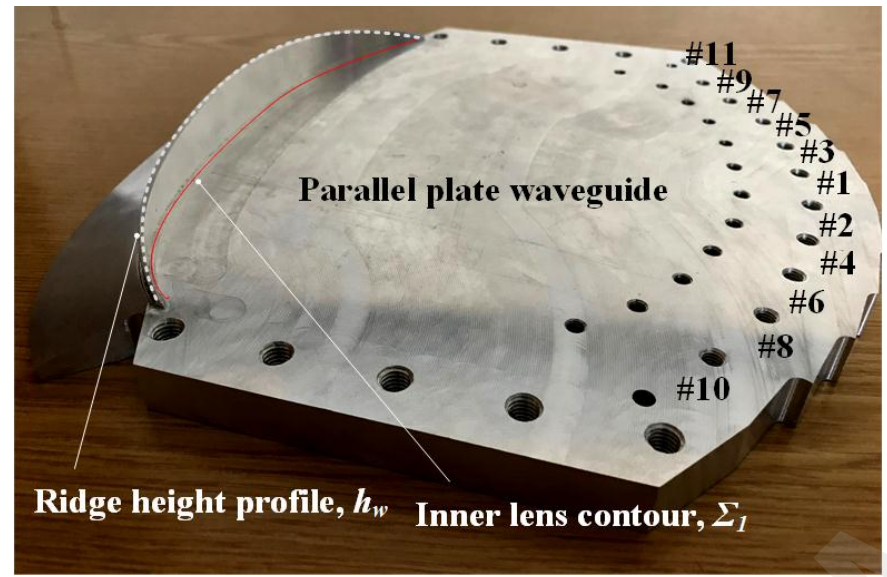

(b)

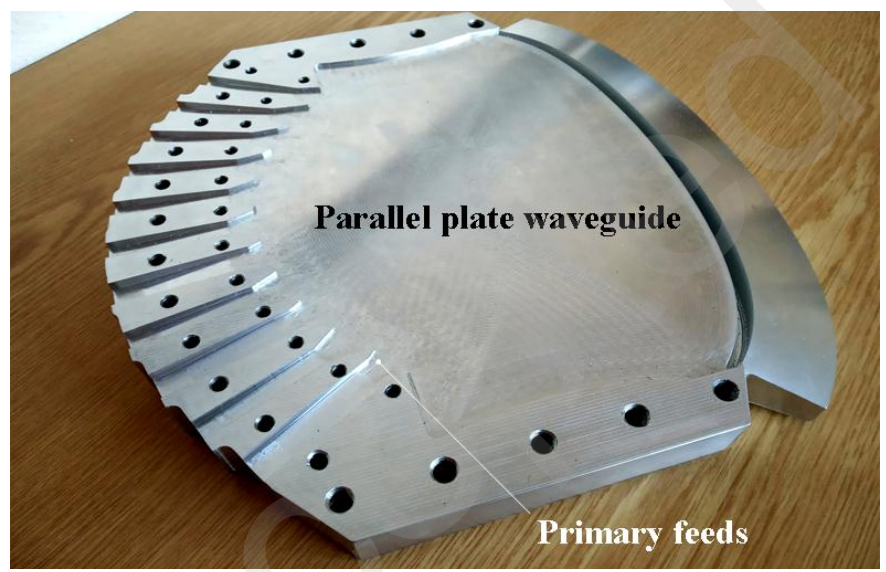

(c)

Fig 8. Antenna prototype. (a) 3D view, (b) Transversal blade part and (c) transversal cavity part

level. The feeds distributions of both lenses are detailed in Fig. $6 \mathrm{c}$, illustrating clearly the way the $3^{\circ}$ spacing between the 22 beams is achieved. This approach is similar to the one proposed in [22] for the first stack (level 1) of Rotman lenses, where a 2D stack of Rotman lenses is arranged to produce a hexagonal beam lattice.

A pattern-based optimization, as presented in section II, has been implemented to achieve the desired performance at the central frequency $f_{0}=30 \mathrm{GHz}$, for the extreme beam

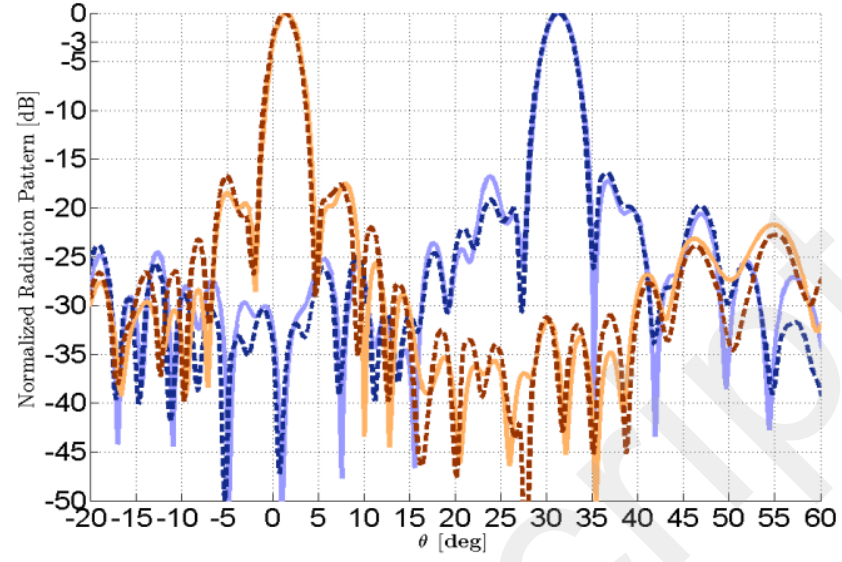

(a)

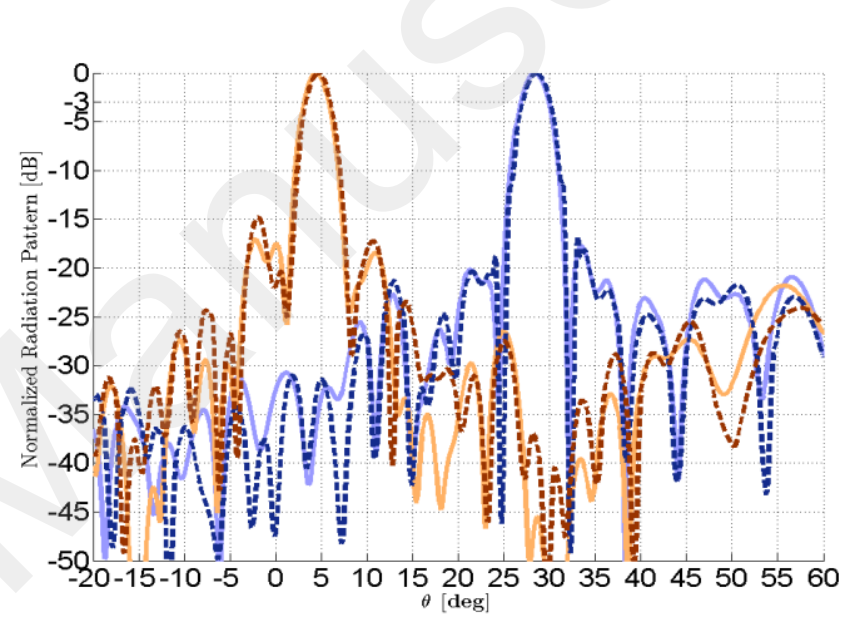

(b)

Fig 9. H-plane normalized radiation patterns of the PPW beamformer at $f_{0}=30 \mathrm{GHz}$. (a) Lens 1 - feeds \#11 $\left(\theta_{1}=31.5^{\circ}\right)$,\#1 $\left(\theta_{1}=1.5^{\circ}\right)$. (b) Lens 2 - feeds \#10 $\left(\theta_{1}=28.5^{\circ}\right)$, \#2 $\left(\theta_{1}=4.5^{\circ}\right)$. Final FEM simulations (continuous line) and measurements (dotted line).

pointing at $\theta_{2}=\theta_{1}=31.5^{\circ}$. The polynomial coefficients resulting from the optimization process are the following: $p=[-0.217,-0.747,-0.165,-0.090,-0.246,0]$ and $q=[-0.527,-0.048,0.038,0.061,-0.237,0]$. Then the parameter $g$ is tuned to achieve the best results over the complete scanning range under consideration, leading to $g=1.1$.

The numerical results obtained with the GO model for the extreme and central beams associated to both lenses (Lens 1 and 2, Fig. 6) are plotted in Fig. 7a and 7b respectively. They are compared to the HFSS results obtained with the final FEM design where PEC boundaries are defined, as in the final prototype. The choice to use metallic sidewalls in the prototype leads to a simpler design and assembly (no absorbing material required) but slightly degrades side lobes away from the main lobe.

A first comparison is provided for the feed defined at $\theta_{1}=31.5^{\circ}$ (Fig. 7a), where the accuracy of the GO model (red continuous line) is confirmed by the excellent agreement with the FEM simulation (blue dotted line). The main beam 


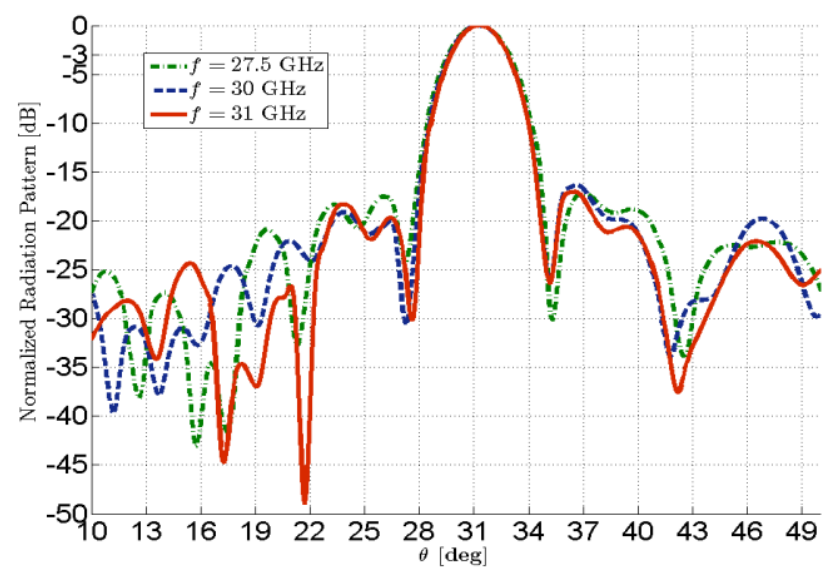

(a)

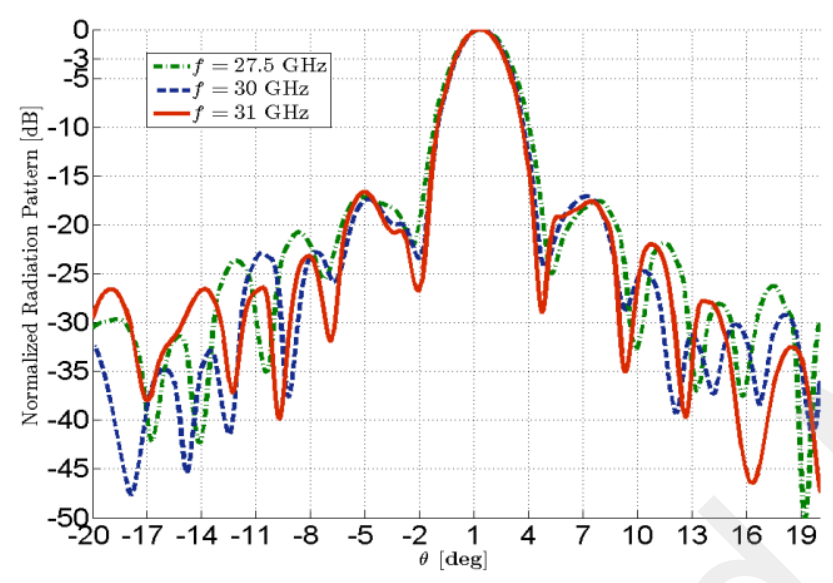

(b)

Fig 10. Measured H-plane normalized radiation patterns of the PPW beamformer for the manufactured Lens $1-$ (a) feed \#11 $\left(\theta_{1}=31.5^{\circ}\right)$ and (b) feed \#1 $\left(\theta_{1}=1.5^{\circ}\right)$ at three frequencies $f=27.5,30$ and $31 \mathrm{GHz}$.

direction and the HPBW computed with the GO and FEM models are equal to $31.5^{\circ}, 3.1^{\circ}$ and $31.3^{\circ}, 3.1^{\circ}$ respectively. The first side lobe level equals -16 and $-16.8 \mathrm{~dB}$ for both models. Some higher side lobes are observed far away from the main lobe region $\left(\left[45^{\circ}, 60^{\circ}\right]\right)$, explained by the reflection effects due to the metallic side walls. Those effects remain quite low, limited to power levels $20 \mathrm{~dB}$ below the peak directivity. For the central beam $\theta_{1}=1.5^{\circ}$ (Fig. 7a), the prediction given by the GO model (red continuous line) is still in good accordance with the FEM simulation (orange dotted line). The main beam direction and HPBW are identical $\left(1.5^{\circ}\right.$, $2.6^{\circ}$ ) while the side lobe level equals $-17 \mathrm{~dB}$ and $-17.5 \mathrm{~dB}$, respectively for the GO tool and the FEM simulation. The beams associated to feeds \#10 $\left(\theta_{1}=28.5^{\circ}\right)$, \#2 $\left(\theta_{1}=4.5^{\circ}\right)$ for Lens 2 (Fig. 6c) are plotted in Fig. 7b. As previously observed in Fig. 7a for Lens 1, the GO and HFSS results are in very good agreement. Higher side lobe levels are also observed for the extreme feed \#10, explained by the metallic side walls implemented, without major impact on the validation proposed here. Hence, these results support the

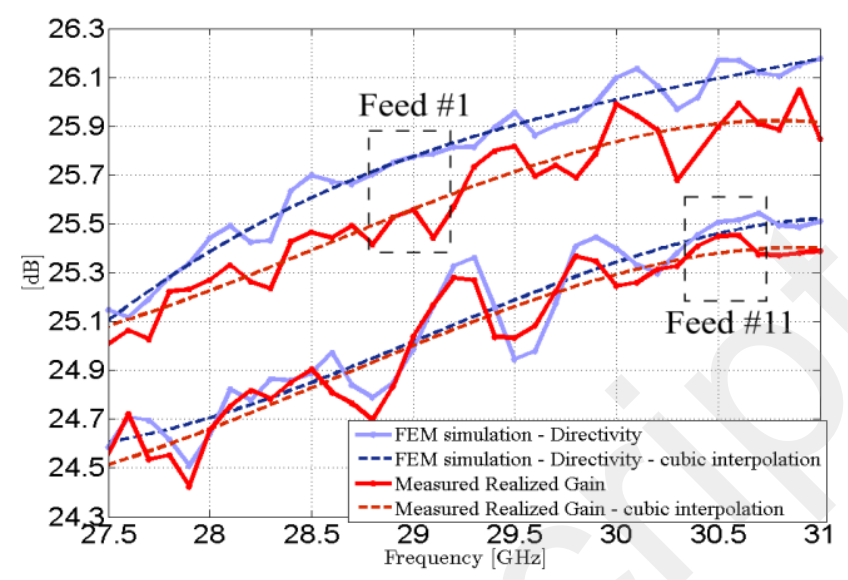

Fig 11. Measured realized gain (red curve) and simulated directivity (blue curve) of of the PPW beamformer for feeds \#1 $\left(\theta_{1}=1.5^{\circ}\right)$ and \#11 $\left(\theta_{1}=\right.$ $\left.31.5^{\circ}\right)$ over the entire Ka-downlink frequency band $([27.5-31] \mathrm{GHz})$.

manufacturing of the simpler prototype with metallic walls on the edges of the PPW section.

\section{B. Experimental results}

The antenna design described in the previous section has been manufactured in bare aluminum. As a $180^{\circ}$ rotational symmetry is applied to obtain the final two-lens antenna system (Fig. 6b), only one lens is manufactured and measured in this experimental validation. The prototype is composed of two blocks corresponding to the top and bottom parts of the antenna as defined in Fig. 1a. These blocks are assembled by means of screws, as shown in Fig. 8a. The transversal ridge part (or transversal blade) is presented in Fig. 8b, where we can recognize both the inner lens $\left(\Sigma_{1}\right)$ and the ridge height profiles $\left(h_{w}\right)$, the transversal cavity bloc is shown in Fig. 8c. Each part has been manufactured using a conventional milling process. The prototype has been measured using the far-field facility available at IETR. The main objective of the test campaign was to evaluate the radiation pattern along the lens focusing plane ( $x O y$ plane in Fig. 1a) to validate the numerical tool and the comparisons provided in Fig. 7.

Comparisons between the final FEM simulation results and the measurements are reported in Fig. 9 for the extreme and central beams of both lenses. An excellent agreement is obtained along the scanning range and for power levels down to $30 \mathrm{~dB}$ below the peak directivity. The agreement is maintained over the entire scanning range $\left[-90^{\circ} 90^{\circ}\right]$ acquired during measurements (not shown here for readability and brevity reasons). For the extreme feed \#11 of Lens 1 (Fig. 9a), the main beam directions equal $31.3^{\circ}$ and $31.2^{\circ}$ respectively for the FEM simulation and the measurements. The HPBW $\theta_{3 d B}$ obtained are identical while the SLL are equal to -16.8 and $-16.4 \mathrm{~dB}$ respectively. For the central feed \#1 (Fig. 9a), the pointing angle, HPBW and SLL equal $1.5^{\circ}, 2.6^{\circ},-17.5 \mathrm{~dB}$ and $1.3^{\circ}, 2.6^{\circ},-16.7 \mathrm{~dB}$ respectively for the FEM simulation and the measurements. The comparison given for the second lens (feeds \#10 and \#2) in Fig. 9b also confirms further the excellent agreement observed between simulation and measurement.

The radiation patterns measured in H-plane at lower (27.5 $\mathrm{GHz})$, central $(30 \mathrm{GHz})$ and upper $(31 \mathrm{GHz})$ frequencies are 


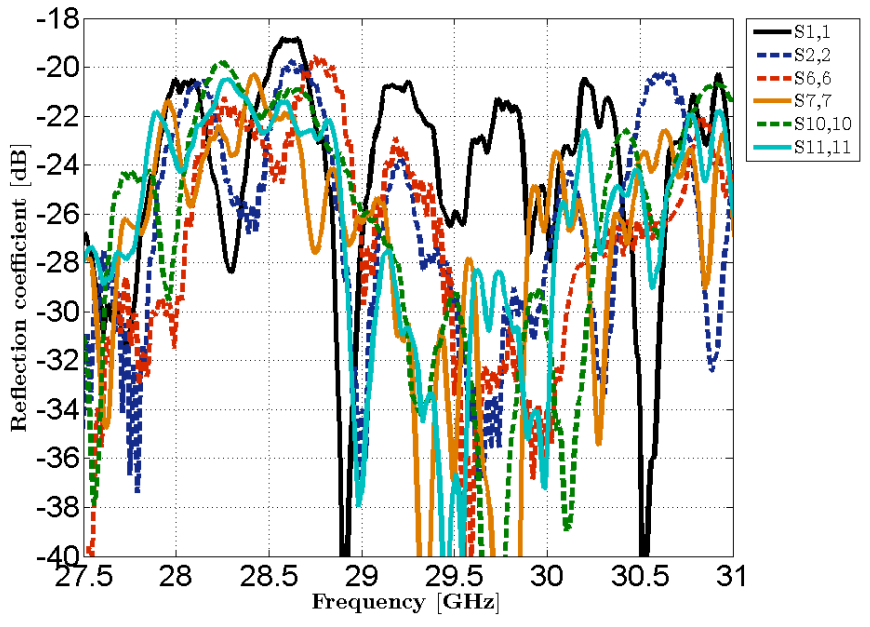

(a)

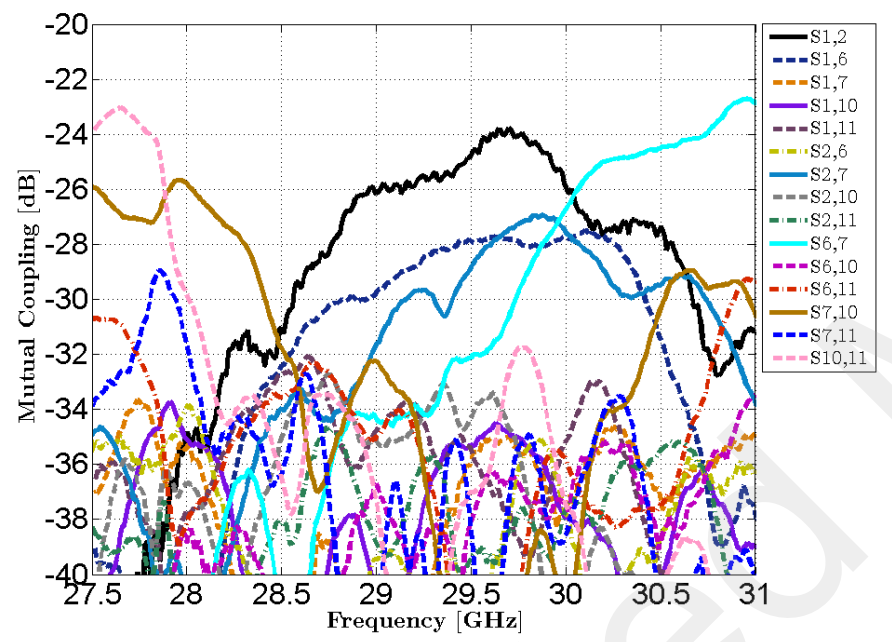

(b)

Fig 12. Measured S-parameters of the PPW beamformer over the entire Ka down-link frequency band ([27.5-31] GHz), (a) reflection and (b) mutual coupling coefficients.

reported in Fig. 10 for the extreme $\left(\theta_{1}=31.5^{\circ}\right)$ and central $\left(\theta_{1}=31.5^{\circ}\right)$ beams. This figure confirms that the radiation properties (pointing angle, HPBW and SLL) are preserved along the frequency band. The HPBW measured equal $3.3^{\circ}, 3^{\circ}$ and $2.9^{\circ}, 2.6^{\circ}$ at both lower and upper frequencies, respectively for feed \#11 and feed \#1.

The measured realized gain values are compared to the directivity computed with the FEM simulation [23] in Fig. 11 for the extreme $\left(\theta_{1}=31.5^{\circ}\right)$ and central $\left(\theta_{1}=1.5^{\circ}\right)$ feeds. Low scanning loss is expected at the central frequency $f_{0}=30 \mathrm{GHz}$, where the computed directivities equal $26 \mathrm{~dB}$ and $25.3 \mathrm{~dB}$ respectively for feeds \#1 and \#11. This has been confirmed with measured realized gain values equal to 25.8 $\mathrm{dB}$ for feed \#1, corresponding to a radiation efficiency $\eta=95.5 \%$, and $25.2 \mathrm{~dB}(\eta=97.7 \%)$ for feed \#11. The measured input reflection coefficients are better than $-19 \mathrm{~dB}$ for feeds \#1, \#2, \#6, \#7, \#10 and \#11, as shown in Fig. 12a;

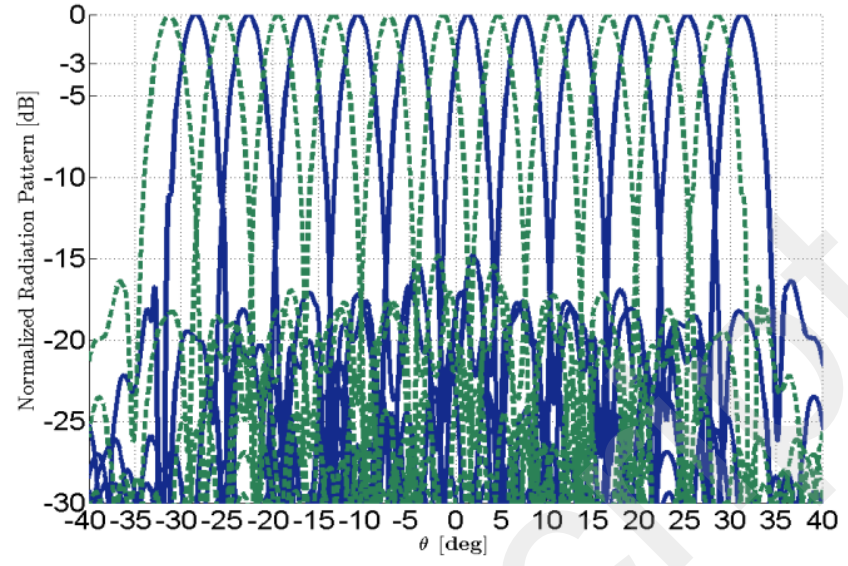

Fig. 13. Measured H-plane normalized radiation patterns of the final PPW beamformer design at $f_{0}=30 \mathrm{GHz}$ for all the feeds (continuous line) and for the same beamforer considering the $180^{\circ}$ rotation around $\mathrm{R}_{\mathrm{L}}$ (dash line).

they are in line with the measured radiation efficiency. Mutual coupling coefficients measured between those feeds are limited to $-22 \mathrm{~dB}$ (Fig. 12b). For readability and brevity purposes, the measured reflection and mutual coupling coefficients associated to the other feeds are not presented here, but are also maintained respectively below -19 and -22 dB.

The complete coverage with the 22 measured beams is shown in Fig. 13. The patterns obtained at $f_{0}=30 \mathrm{GHz}$ with the considered lens (continuous blue line) have been combined with the ones obtained after mirroring along axis $\mathrm{R}_{\mathrm{L}}$, as shown in Fig. 6a. As observed, a $-3 \mathrm{~dB}$ cross-over level is obtained while maintaining low side lobe levels over the entire scanning range $\left(\left[-31.5^{\circ} 31.5^{\circ}\right]\right)$.

\section{CONCLUSION}

This paper described a continuous PPW delay lens with polynomial inner/outer lens contours and ridge height profile. A dedicated analytical model, based on ray tracing, was updated to take into account such profiles and compared with previously reported beamformers. The numerical results confirm the potential of the proposed profiles with RMS values of normalized path length errors very similar to those of simplified constrained lens models over a large scanning range $\left(\left[\begin{array}{ll}-30^{\circ} & 30^{\circ}\end{array}\right]\right)$. The corresponding radiated patterns present reduced and more balanced side lobe levels, when compared to the previously introduced solution using elliptical contour and profile for the delay-lens shape.

An experimental validation has been proposed over the down-link Ka-band (27.5-31 GHz), confirming the properties in scanning offered by the concept. Excellent beam performance (HPBW, SLL) are obtained over a wide angular range $\left[-31.5^{\circ}, 31.5^{\circ}\right]$, including low scanning loss and high radiation stability within the overall frequency band. High radiation efficiencies are demonstrated with this fully metallic design, particularly suitable for space applications. The mechanical simplicity offered by the concept is also quite attractive to propose low-cost multi-beam satellite platforms. 
Future investigations on this concept will include efforts for height reduction, which is of interest when a stack of such beamformers is needed to produce a planar array antenna. Alternative radiation properties, such as shaped or defocused patterns need also to be further investigated.

\section{ACKNOWLEDGEMENT}

This works has been supported by the European Space Agency (ESA) and Thales Alenia Space - France in the frame of ESA's networking partnering initiative (NPI) and also by the European Union through the European Regional Development Fund (ERDF), and by Ministry of Higher Education and Research, Brittany and Rennes Métropole, through the CPER Project SOPHIE / STIC \& Ondes

\section{REFERENCES}

[1] P. Bosshard et al., "Thales Alenia Space HTS/VHTS Multiple beam antennas sub-systems on the right track," in Proc. 10th Eur. Conf. Antennas Propag. (EUCAP), Davos, Switzerland, pp. 1-5, Apr. 2016.

[2] B. Palacin et al., "Multibeam antennas for very high throuput satellites in Europe: Technologies and trends," in Proc. 11th Eur. Conf. Antennas Propag. (EUCAP), Paris, France, pp. 2413-2417, Mar. 2017.

[3] W. Rotman, "Wide-angle scanning with microwave double-layer pillboxes," IRE Trans. Antennas Propag., vol. 6, no. 1, pp. 96-105, Jan. 1958.

[4] E. L. Holzman, "Pillbox antenna design for millimeter-wave base-station applications," IEEE Antennas Propag. Mag., vol. 45, no. 1, pp. 27-37, Feb. 2003.

[5] M. Ettorre, R.Sauleau, and L. Le Coq, "Multi-beam multi-layer leakywave SIW pillbox antenna for millimeter-wave applications," IEEE Trans. Antennas Propag., vol. 59, no. 4, pp. 1093-1100, Apr. 2011.

[6] M. Ettorre, F. F. Manzillo, M. Casaletti, R.Sauleau, L. Le Coq and N. Capet,"Continuous transverse stub array for Ka-band applications," IEEE Trans. Antennas Propag., vol. 63, no. 11, pp. 4792-4800, Nov. 2015.

[7] J. Ruze, "Wide-angle metal-plate optics," IRE Proc., vol. 38, no. 1, pp. 53-59, Jan. 1950.

[8] W. Rotman and R. Turner, "Wide-angle microwave lens for line source applications," IEEE Trans. Antennas Propag., vol. 11, no. 6, pp. 623632, Nov. 1963.

[9] A. F. Peterson and E. O. Rausch, "Scattering matrix integral equation analysis for the design of a waveguide Rotman lens," IEEE Trans. Antennas Propag, vol. 47, no. 5, pp. 870-878, May 1999.

[10] R. Luneberg, Mathematical theory of optics. Providence, 1944, vol. 1.

[11] C. D. Diallo, E. Girard, H. Legay, and R. Sauleau, "All-metal Ku-band Luneburg lens antenna based on variable parallel plate spacing fakir bed of nails," in Proc. $11^{\text {th }}$ Eur. Conf. Antennas Propag. (EUCAP), Paris, France,pp. 1401-1404, Mar. 2017

[12] O. Quevedo-Teruel, J. Miao, M. Mattsson, A. Algaba-Brazalez, M. Johansson, and L. Manholm, "Glide-symmetric fully metallic Luneburg lens for 5G communications at Ka-Band," IEEE Antennas and Wireless Propag. Lett., vol. 17, no. 9, pp. 1588-1592, Sept. 2018.

[13] R. F. Rinehart, "A solution of the problem of rapid scanning for radar antennas," Journal of Applied Physics, vol. 19, pp. 860-862, Oct. 1948.

[14] Q. Liao, N. J. G. Fonseca, and O. Quevedo-Teruel, "Compact multibeam fully metallic geodesic Luneberg lens based on non-euclidian transformation optics," IEEE Trans. Antennas Propag., vol. 66, no. 12, pp. $7383-7388$, Dec. 2018

[15] H. Legay, S. Tubau, E. Girard, J.-Ph. Fraysse, R. Sauleau, M. Ettorre, and N. J. G. Fonseca, "Multiple beam antenna based on a parallel plate waveguide continuous delay lens beamformer," in Proc. Int. Symp. Antennas Propag., pp. 118-119, 2016, Okinawa, Japan, Oct. 2016.

[16] F. Doucet, N.J.G. Fonseca, E. Girard, H. Legay, and R. Sauleau, "Analysis and design of a continuous parallel plate waveguide multiple beam lens antenna at Ku-band," in Proc. 11h Eur. Conf. Antennas Propag. (EUCAP), Paris, France, pp. 3631-3635, Mar. 2017.
[17] F. Doucet, N.J.G. Fonseca, E. Girard, H. Legay, and R. Sauleau, "Analytical model and study of continuous parallel plate waveguide lens-like multiple beam antennas," IEEE Trans. Antennas Propag., vol. 66, no. 9, pp. 4426-4436, Aug. 2018.

[18] F. Doucet, N.J.G. Fonseca, E. Girard, H. Legay, and R. Sauleau, "Comparison of optimization procedures for the design of continuous parallel plate waveguide multiple beam lens antennas," in Proc. 10th Eur. Conf. Antennas Propag. (EUCAP), London, United Kindom, pp. 15, Apr. 2018.

[19] N.J.G. Fonseca, H. Legay, S. Tubau, J. P. Fraysse, E. Girard, M. Ettorre, and R. Sauleau, "Continuous parallel plate waveguide beamformer based on a bifocal constrained lens design," in Proc. IEEE Int. Symp. Antennas Propag., pp. 1347-1348, Fajardo, Porto Rico, Jun. 2016.

[20] D. S. Weile and E. Michielssen, "Genetic algorithm optimization applied to electromagnetics : a review," IEEE Trans. Antennas Propag., vol. 45, no. 3, pp. 343-353, Mar. 1997.

[21] G. Godi, R. Sauleau, L. Le Coq, and D. Thouroude, "Design and optimization of three-dimensional integrated lens antennas with genetic algorithm," IEEE Trans. Antennas Propag., vol. 55, no. 3, pp. 770-775, Jan. 2007.

[22] M. J. Maybell, G. S. Hardie, "EHF waveguide Rotman lens for reconfigurable SATCOM," in Proc. 39th ESA Antenna Workshop on Multibeam and Reconfigurable Antennas for Space Applications, Noordwijk, The Netherlands, Oct. 2018.

[23] Ansoft HFSS version 18.0, 1984-2017, Ansoft Corporation. 
